\title{
Quantum criticality and universal scaling of strongly attractive spin-imbalanced Fermi gases in a one-dimensional harmonic trap
}

\author{
Xiangguo Yin, ${ }^{1}$ Xi-Wen Guan, ${ }^{2, *}$ Shu Chen, ${ }^{1}$ and Murray T. Batchelor ${ }^{2,3}$ \\ ${ }^{1}$ Institute of Physics, Chinese Academy of Sciences, Beijing 100190, China \\ ${ }^{2}$ Department of Theoretical Physics, Research School of Physics and Engineering, Australian National University, \\ Canberra ACT 0200, Australia \\ ${ }^{3}$ Mathematical Sciences Institute, Australian National University, Canberra ACT 0200, Australia
}

(Received 18 January 2011; published 13 July 2011)

\begin{abstract}
We investigate quantum criticality and universal scaling of strongly attractive Fermi gases confined in a one-dimensional harmonic trap. We demonstrate from the power-law scaling of the thermodynamic properties that current experiments on this system are capable of measuring universal features at quantum criticality, such as universal scaling and Tomonaga-Luttinger liquid physics. The results also provide insights on recent measurements of key features of the phase diagram of a spin-imbalanced atomic Fermi gas [Y. Liao et al., Nature (London) 467, 567 (2010)] and point to further study of quantum critical phenomena in ultracold atomic Fermi gases.
\end{abstract}

DOI: 10.1103/PhysRevA.84.011602

PACS number(s): 67.85.-d, 03.75.Hh, 03.75.Ss, 05.30.Rt

Very recently, the one-dimensional (1D) strongly attractive two-component Fermi gas has attracted much attention from theorists [1-7] and experimentalists [8] due to the existence of an exotic pairing mechanism. Investigation [9-11] shows that this novel pairing is closely related to the elusive FuldeFerrell-Larkin-Ovchinnikov (FFLO) [12] state involving BCS pairs with nonzero center-of-mass momenta.

The 1D Fermi gas is one of the most important exactly solvable quantum many-body systems. It was solved long ago by Yang [13] and Gaudin [14] using the Bethe ansatz. Although the study of the attractive Fermi gas was initiated soon after $[15,16]$, it was not until much later that this model began to receive more attention [17]. In terms of the polarization $p$, the model exhibits three quantum phases at zero temperature [1-3]: the fully paired phase which is a quasicondensate with $P=0$, the fully polarized normal Fermi liquid with $P=1$, and the partially polarized (1D FFLO-like) phase for $0<$ $P<1$.

For a trapped imbalanced Fermi gas it is found [1,2] within the local density approximation (LDA) that a partially polarized 1D FFLO-like state sits in the trapping center surrounded by wings composed of either a fully paired state or a fully polarized Fermi gas. The phase boundaries of the zero-temperature phase diagram shown in Fig. 1 can be determined precisely from the exact solution by the vanishing of the axial density difference (solid line) and the minority state axial density (dashed line). The key features of this $T=0$ phase diagram were experimentally confirmed using finite temperature density profiles of trapped fermionic ${ }^{6} \mathrm{Li}$ atoms [8].

Most recently, quantum criticality and universal scaling behavior [18] are being explored in low-dimensional cold atomic matter, for example, in experiments on the twodimensional Bose gas [19], following a theoretical scheme for mapping out quantum criticality [20,21]. From this viewpoint, the 1D imbalanced Fermi gas, exhibiting novel

\footnotetext{
*xwe105@physics.anu.edu.au
}

phase transitions at $T=0$, is particularly valuable to test universal scaling through finite temperature density profiles of trapped ultracold atoms [22]. Here we illustrate that finitetemperature properties of the quasi-1D trapped Fermi gas allow the exploration of a wide range of physical phenomena, such as universal Tomonaga-Luttinger liquid (TLL) physics, scaling theory, and the nature of the FFLO state at quantum criticality.

We consider the 1D $\delta$-interacting attractive spin- $1 / 2$ Fermi gas with $N=N_{\uparrow}+N_{\downarrow}$ fermions of mass $m$ and external magnetic field $H$. The system is described by the Hamiltonian

$$
\begin{aligned}
\mathcal{H}= & -\frac{\hbar^{2}}{2 m} \sum_{i=1}^{N} \frac{\partial^{2}}{\partial x_{i}^{2}}+g_{1 \mathrm{D}} \sum_{i=1}^{N_{\uparrow}} \sum_{j=1}^{N_{\downarrow}} \delta\left(x_{i}-x_{j}\right) \\
& -\frac{1}{2} H\left(N_{\uparrow}-N_{\downarrow}\right),
\end{aligned}
$$

in which the three terms are kinetic energy, interaction energy, and Zeeman energy, respectively. Here the intercomponent interaction is related to an effective $1 \mathrm{D}$ scattering length $g_{1 \mathrm{D}}=$ $-\frac{2 \hbar^{2}}{m a_{1 \mathrm{D}}}$, which can be tuned from the weakly interacting regime $\left(g_{1 \mathrm{D}} \rightarrow 0^{-}\right)$to the strong-coupling regime $\left(g_{1 \mathrm{D}} \rightarrow-\infty\right)$ via Feshbach resonances and optical confinement. For convenience, we define the interaction strength as $c=m g_{1 \mathrm{D}} / \hbar^{2}$ and dimensionless parameter $\gamma=c / n$ for physical analysis, where $n=N / L$ is the linear density and $L$ is the length of the system. We set the Boltzmann constant $k_{B}=1$.

For the strongly attractive spin-1/2 Fermi gas at finite temperatures, the thermodynamics of the homogeneous system is described by two coupled Fermi gases of bound pairs and excess fermions in the charge sector and ferromagnetic spin-spin interaction in the spin sector. Spin fluctuations are suppressed by a strong effective magnetic field at low temperatures. For the physically interesting low-temperature and strong-coupling regime, i.e., $T \ll \epsilon_{b}, H$ and $\gamma \gg 1$, a high-precision equation of state [22-24] can be derived from the thermodynamic Bethe ansatz equations [25] in terms of the Yang-Yang grand canonical ensemble [26]. Using the 


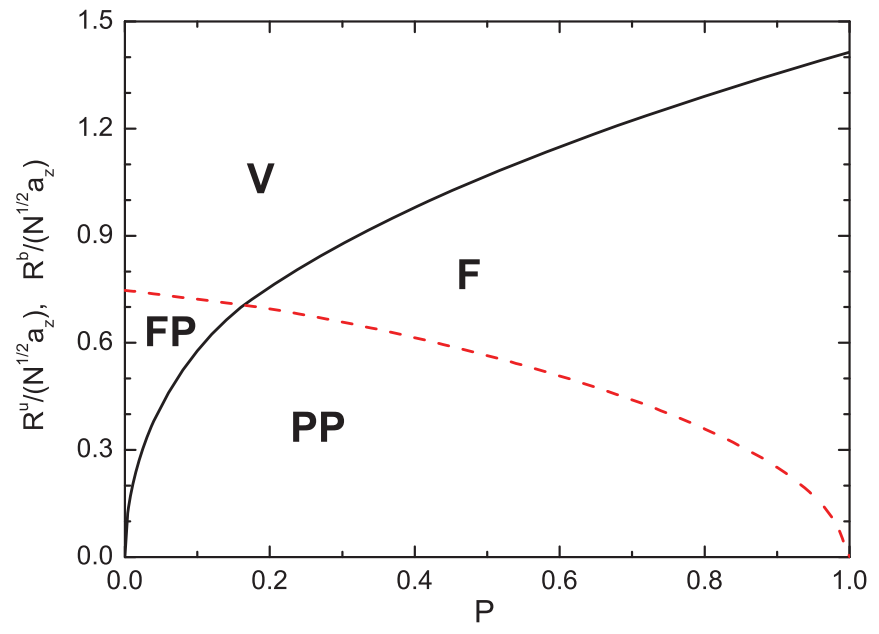

FIG. 1. (Color online) Phase diagram for two-component fermions in a harmonic trap at zero temperature as a function of polarization with $N a_{1 \mathrm{D}}^{2} / a_{z}^{2}=0.2$. Here FP denotes the fully paired phase, $\mathrm{F}$ the fully unpaired phase, and PP the FFLO-like phase. $\mathrm{V}$ is the vacuum phase.

binding energy $\varepsilon_{b}=\hbar^{2} c^{2} / 4 m$ as the unit of energy and defining $\tilde{\mu}=\mu / \varepsilon_{b}, h=H / \varepsilon_{b}, t=T / \varepsilon_{b}$, and $\tilde{p}=p /\left|c \varepsilon_{b}\right|$, the pressure $\tilde{p}=\tilde{p}^{b}+\tilde{p}^{u}$ of the system is found to be

$$
\begin{gathered}
\tilde{p}^{b}=-\frac{t^{3 / 2} f_{3 / 2}^{b}}{2 \sqrt{\pi}}\left(1-\frac{t^{3 / 2} f_{3 / 2}^{b}}{16 \sqrt{\pi}}-\frac{t^{3 / 2} f_{3 / 2}^{u}}{\sqrt{2 \pi}}\right), \\
\tilde{p}^{u}=-\frac{t^{3 / 2} f_{3 / 2}^{u}}{2 \sqrt{2 \pi}}\left(1-\frac{t^{3 / 2} f_{3 / 2}^{b}}{\sqrt{\pi}}\right),
\end{gathered}
$$

where $f_{n}^{b}=\operatorname{Li}_{n}\left(-e^{A_{b} / t}\right)$ and $f_{n}^{u}=\operatorname{Li}_{n}\left(-e^{A_{u} / t}\right)$ in terms of the standard polylogarithm function $\operatorname{Li}_{n}(x)$, with

$$
\begin{gathered}
A_{b}=2 \tilde{\mu}+1-\tilde{p}^{b}-4 \tilde{p}^{u}-\frac{t^{5 / 2} f_{5 / 2}^{b}}{16 \sqrt{\pi}}-\sqrt{\frac{2}{\pi}} t^{5 / 2} f_{5 / 2}^{u}, \\
A_{u}=\tilde{\mu}+\frac{h}{2}-2 \tilde{p}^{b}-\frac{t^{5 / 2} f_{5 / 2}^{b}}{2 \sqrt{\pi}}+f_{s} .
\end{gathered}
$$

Here $\quad f_{s}=t e^{-h / t} e^{-2 \tilde{p}^{u} / t} I_{0}\left(2 \tilde{p}^{u} / t\right) \quad$ and $\quad I_{n}(x)=$ $\sum_{k=0}^{\infty} \frac{1}{k !(n+k) !}(x / 2)^{n+2 k}$. The thermodynamic quantities such as the particle density $n, n^{u}$ for unpaired fermions, $n^{b}$ for paired fermions, and compressibility follow from Eqs. (2) and the standard thermodynamic relations. The total pressure serves as the equation of state which provides high-precision thermodynamics over a wide temperature range $T<0.2 \varepsilon_{b}$ (see [22,23]).

For spin-imbalanced attractive fermions in a harmonic trap, the equation of state (2) can be reformulated within the LDA by the replacement $\mu(z)=\mu(0)-\frac{1}{2} m \omega_{z}^{2} z^{2}$ in which $z$ is the position and $\omega_{z}$ is the frequency within the trap. Using the dimensionless chemical potential, this becomes $\tilde{\mu}(z)=\tilde{\mu}(0)-2 \tilde{z}^{2}$, where $\tilde{z}=z /\left(a_{z}^{2}|c|\right)$ with the harmonic characteristic radius $a_{z}=\sqrt{\hbar /\left(m \omega_{z}\right)}$. The total particle num- ber $N=\int_{-\infty}^{\infty} n(z) d z$ and polarization $P=\int_{-\infty}^{\infty} n^{u}(z) d z / N$ become

$$
\begin{gathered}
N a_{1 \mathrm{D}}^{2} / a_{z}^{2}=4 \int_{-\infty}^{\infty} \tilde{n}(z) d \tilde{z}, \\
P=4 \int_{-\infty}^{\infty} \tilde{n}^{u}(z) d \tilde{z} a_{z}^{2} /\left(N a_{1 \mathrm{D}}^{2}\right),
\end{gathered}
$$

where $\tilde{n}(z)=1 /|\gamma(z)|$ and $\tilde{n}^{u}(z)=n^{u}(z) /|c|$.

At finite temperatures, the phase boundaries can be determined from the equation of state (2) within the LDA (4), where the boundaries of vanishing density difference (black solid line) and vanishing unpaired fermions (red dashed line) form three phases (see Fig. 1). As $t \rightarrow 0$, the phase boundaries determined via Eqs. (2) are consistent with the zero-temperature results. For strong attraction with polarization, the atoms with opposite spin states form hard-core bosons which are strongly correlated with excess fermions. The polarization can be changed by tuning the effective magnetic field. This results in two extreme phases: the fully paired and fully polarized phases. At zero temperature, these phases intersect at global polarization $P=P_{c}$, where we find

$$
P_{c}=\frac{1}{5}\left(1-\frac{64}{75|\gamma|}\right)+O\left(1 / \gamma^{2}\right) \text {. }
$$

Here the phase boundaries are sharp lines and $P_{c}$ is the intersection point in Fig. 1. At finite temperatures the intersection point $P_{c}$ decreases as the temperature increases. This result is consistent with experimental observation [8]. However, for the experimental temperature $t=175 \mathrm{nK}$ (about $0.0267 \varepsilon_{b}$ ), our theoretical intersection point is smaller than the experimentally estimated value. This discrepancy originates merely in defining the edge of the cloud in the experiment where the phase boundaries are no longer sharp lines [27]. We also find that increasing temperature results in shrinking the fully paired phase and populating the vacuum. The existence of these quantum phases at low temperatures is a manifestation of different TLL phases, i.e., a two-component TLL of FLLO-like states and a TLL of hard-core bosons and a TLL of unpaired fermions [23], where the low-lying excitations are close to the Fermi points.

The FFLO-like state behaves like a mixture of composite fermions with mass $2 m$ and excess fermions with mass $m$ [22]. The density of state changes critically as the driving parameter chemical potential is varied across the phase boundaries. Figure 2 shows the density distributions for $N a_{1 \mathrm{D}}^{2} / a_{z}^{2}=0.2$ at zero temperature and for two different values of polarization. For small polarization (10\%), an FFLO-like state coexists in the trap center with a BCS-like state at the edges, while for large polarization $(50 \%)$, the FFLO-like state lies in the trap center with a fully polarized normal state at the edges. We also find the FFLO-like state occupies the whole trap for the critical polarization $(16 \%)$, which is consistent with the experimental observation [8]. We now demonstrate how to map out the zero-temperature phase diagram and quantum criticality from finite temperature density and compressibility profiles of the trapped gas.

The system exhibits universal scaling behavior in the vicinity of the critical points as a result of the many-body effects. The thermodynamic functions of the homogeneous 


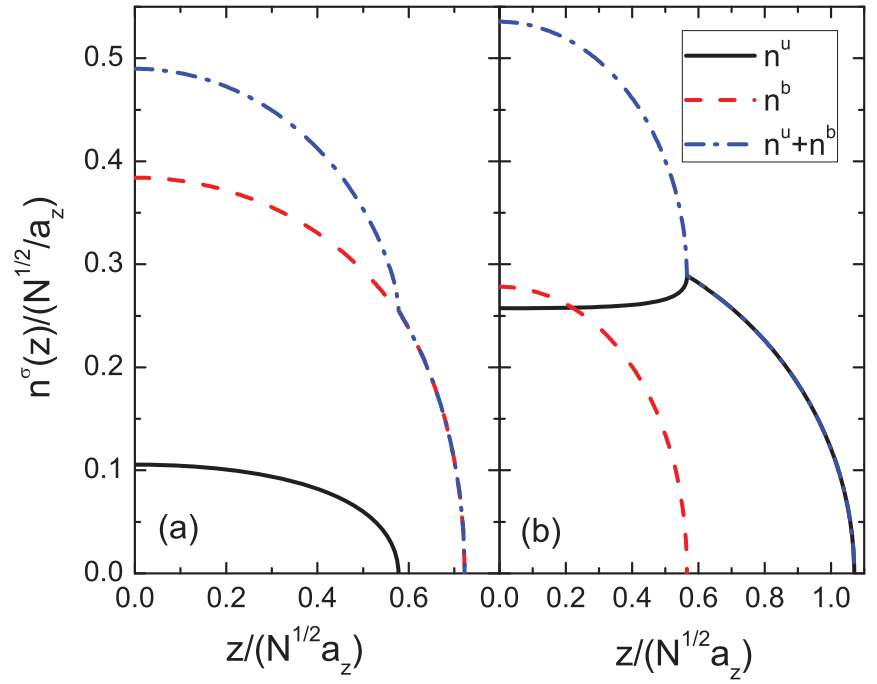

FIG. 2. (Color online) The zero-temperature density distribution profiles for trapped fermions with $N a_{1 \mathrm{D}}^{2} / a_{z}^{2}=0.2$ for (a) $P=10 \%$ and (b) $P=50 \%$. Here we have $n^{u}=n_{\uparrow}-n_{\downarrow}$ and $n^{b}=n_{\downarrow}$.

gas can be cast into a universal scaling form [18,20,21]. For example, the density and compressibility scale as

$$
\begin{aligned}
& n(\mu, T)=n_{0}+T^{\frac{d}{z}+1-\frac{1}{v z}} \mathcal{G}\left(\frac{\mu-\mu_{c}}{T^{\frac{1}{v z}}}\right), \\
& \kappa(\mu, T)=\kappa_{0}+T^{\frac{d}{z}+1-\frac{2}{v z}} \mathcal{F}\left(\frac{\mu-\mu_{c}}{T^{\frac{1}{v z}}}\right) .
\end{aligned}
$$

Here the dimensionality $d=1$, the dynamical critical exponent $z=2$, and the correlation length exponent $v=1 / 2$
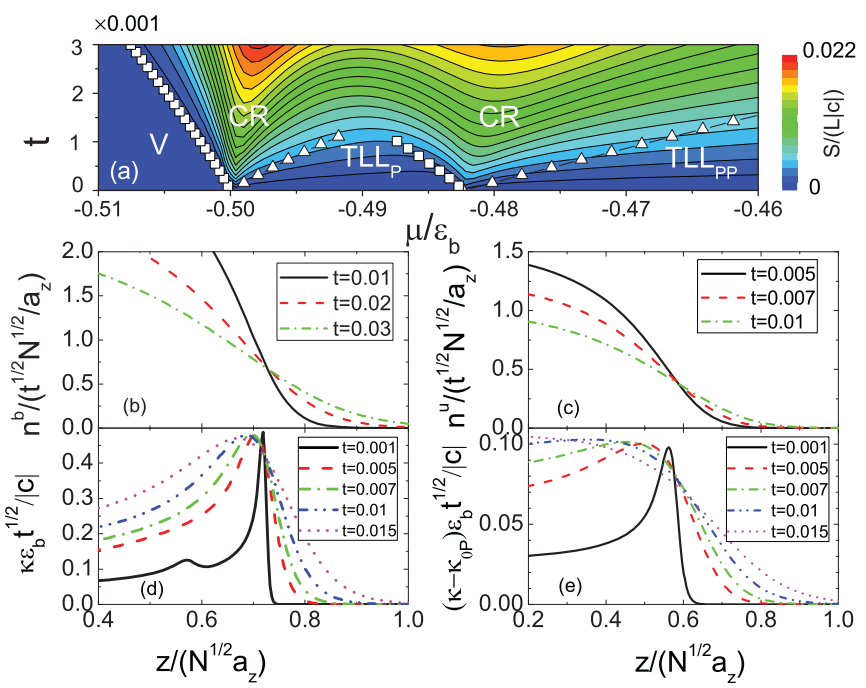

FIG. 3. (Color online) Quantum criticality for low polarization. (a) Contour plot of entropy in the $t$ - $\mu$ plane where $t=T / \varepsilon_{b}$. Diamonds and triangles indicate crossover temperatures separating the quantum critical regimes from vacuum, single-component TLL of paired fermions, and two-component TLLPP of FLLO-like states. The density profiles demonstrate how to locate the critical points (b) $\mu_{c 2}$ and (c) $\mu_{c 4}$ and thus map out their phase boundaries. The corresponding compressibility curves (d) and (e) intersect at the critical points after a subtraction of the background compressibility. for the 1D strongly attractive Fermi gas [22]. The universal scaling functions $\mathcal{G}(x)$ and $\mathcal{F}(x)$ describe quantum and thermal fluctuations at quantum criticality after subtraction of the background density $n_{0}$ and compressibility $\kappa_{0}$.

For small polarization $P<P_{c}$, the chemical potential passes the lower critical point $\mu_{c 2}=-\frac{1}{2}$ from the vacuum into the fully paired phase and then passes the upper critical point $\mu_{c 4} \approx-\frac{h}{2}+\frac{4}{3 \pi}(1-h)^{\frac{3}{2}}+\frac{3}{2 \pi^{2}}(1-h)^{2}$ from the fully paired phase into the FFLO-like phase. At finite temperatures, contour plots of the entropy clearly indicate universal critical behavior near the critical points [see Fig. 3(a)]. The typical V-shaped crossover temperatures separate the quantum critical regimes of the hard-core bosonic TLLP phase and the two-component FLLO-like TLL $L_{P P}$ phase near the critical points $\mu_{c 2}$ and $\mu_{c 4}$, respectively. Except for the leftmost line separating the vacuum from the critical regime, all of the lines can be determined by the breakdown of the TLL, i.e., when the entropy curves deviate from linear $T$-dependent relations for fixed values of $\mu$ and $h$. These boundaries indicate a crossover from linear dispersion into nonrelativistic dispersion rather than phase transitions at finite $T$.

For large polarization $P>P_{c}$, the chemical potential passes the lower critical point $\mu_{c 1}=-h / 2$ from the vacuum into the fully unpaired phase and then passes the upper critical point $\mu_{c 3} \approx-\frac{1}{2}\left[1-\frac{2}{3 \pi}(h-1)^{\frac{3}{2}}-\frac{2}{3 \pi^{2}}(h-1)^{2}\right]$ from the fully unpaired phase into the FFLO-like phase [see Fig. 4(a)]. Here the typical V-shaped crossover temperatures indicate quantum criticality of the largely polarized Fermi gas near the two critical points $\mu_{c 1}$ and $\mu_{c 3}$, respectively.

The zero-temperature phase boundary separating the vacuum from the fully paired phases can be mapped out from the universal scaling behavior of the pair density curves for
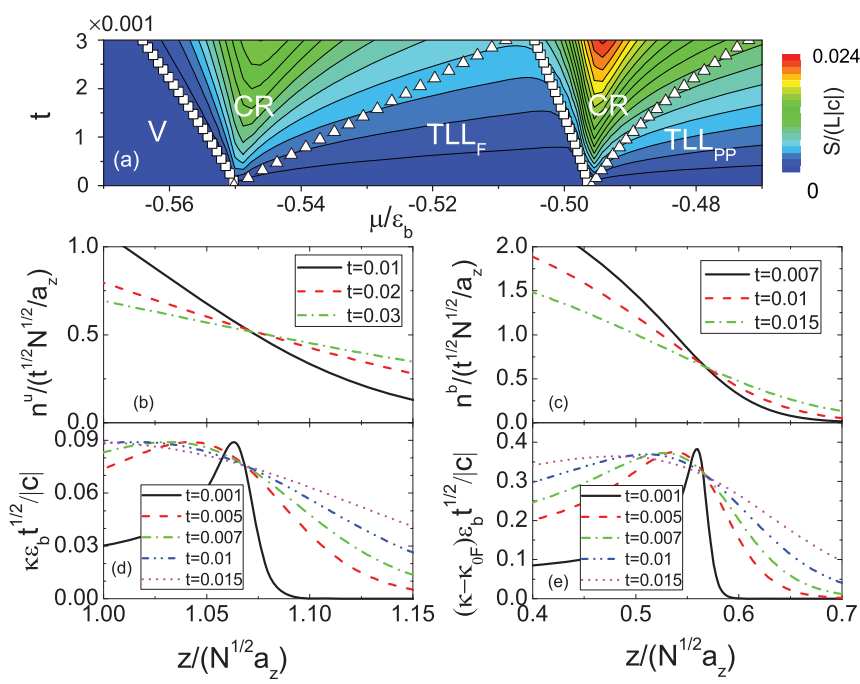

FIG. 4. (Color online) Quantum criticality for high polarization. (a) Contour plot of entropy in the $t-\mu$ plane where $t=T / \varepsilon_{b}$. Diamonds and triangles indicate crossover temperatures separating the quantum critical regimes from vacuum, single-component TLLF of unpaired fermions, and two-component TLLPP of FLLO-like states. The density profiles demonstrate how to locate the critical points (b) $\mu_{c 1}$ and (c) $\mu_{c 3}$ and thus map out their phase boundaries. The corresponding compressibility curves (d) and (e) intersect at the critical points after a subtraction of the background compressibility. 
different temperatures, which intersect at the critical point $\mu_{c 2}$ [see Fig. 3(b)]. Similarly the phase boundary separating the fully paired phase from the FFLO-like phase can be mapped out from the density profiles of unpaired fermions in the trapped gas at different temperatures; i.e., the unpaired density curves for different temperatures intersect at the critical point $\mu_{c 4}$ [see Fig. 3(c)]. Here we have chosen the densities without background near the critical points for practical purposes. We see that the density curves for temperatures below $0.02 \varepsilon_{b}$ well intersect at the critical point. Accordingly, the compressibilities at low temperatures also well intersect at the critical points [see Figs. 3(d) and 3(e)]. Here the background compressibility near $\mu_{c 4}$ is $\kappa_{0 P}=-\frac{2|c|}{\varepsilon_{b} \sqrt{\pi t}} f_{-\frac{1}{2}}^{b}\left(1+\frac{3 \sqrt{t}}{2 \sqrt{\pi}} f_{\frac{1}{2}}^{b}\right)$. The results demonstrate that universal scaling behavior persists in the trapped cloud of atoms [28]. As the temperature decreases, we see that the compressibility evolves a round peak sitting in the phase of the higher density of state. It diverges at zero temperature.
For the high-polarization case, the density profiles of unpaired and paired atoms can be used to map out the phase boundaries $\mu_{c 1}(\mathrm{~V} \rightarrow \mathrm{F})$ and $\mu_{c 3}(\mathrm{~F} \rightarrow \mathrm{PP})$, respectively [see Figs. 4(b) and 4(c)]. Moreover, we show that the corresponding compressibility curves of the trapped gas at low temperatures intersect at the critical points $\mu_{c 1}$ and $\mu_{c 3}$ [see Figs. 3(d) and 3(e)]. Near $\mu_{c 3}$ the background compressibility is $\kappa_{0 F}=$ $-\frac{|c|}{2 \sqrt{2} \varepsilon_{b} \sqrt{\pi t}} f_{-\frac{1}{2}}^{u}$. In a similar fashion, the universal scaling for other thermodynamic quantities such as the specific heat and magnetization are testable through the quantum criticality of the trapped gas. This provides a reliable way to determine quantum phase diagrams and test universal scaling theory and TLL physics in $1 \mathrm{D}$ quantum gases of cold atoms.

This work is supported by the Australian Research Council and by NSF of China and the 973 Projects of MOST. The authors thank T.-L. Ho and E. J. Mueller for stimulating discussions.
[1] G. Orso, Phys. Rev. Lett. 98, 070402 (2007)

[2] H. Hu, X.-J. Liu, and P. D. Drummond, Phys. Rev. Lett. 98, 070403 (2007).

[3] X. W. Guan, M. T. Batchelor, C. Lee, and M. Bortz, Phys. Rev. B 76, 085120 (2007).

[4] M. Casula, D. M. Ceperley, and E. J. Mueller, Phys. Rev. A 78, 033607 (2008).

[5] P. Kakashvili and C. J. Bolech, Phys. Rev. A 79, 041603(R) (2009).

[6] T. Iida and M. Wadati, J. Phys. Soc. Jpn. 77, 024006 (2008).

[7] M. T. Batchelor, A. Foerster, X. W. Guan, and C. C. N. Kuhn, J. Stat. Mech. (2010) P12014.

[8] Y. Liao et al., Nature (London) 467, 567 (2010).

[9] K. Yang, Phys. Rev. B 63, 140511 (2001).

[10] A. E. Feiguin and F. Heidrich-Meisner, Phys. Rev. B 76, 220508 (2007).

[11] E. Zhao and W. V. Liu, Phys. Rev. A 78, 063605 (2008).

[12] P. Fulde and R. A. Ferrell, Phys. Rev. 135, A550 (1964); A. I. Larkin and Yu. N. Ovchinnikov, Sov. Phys. JETP 20, 762 (1965).

[13] C. N. Yang, Phys. Rev. Lett. 19, 1312 (1967).

[14] M. Gaudin, Phys. Lett. A 24, 55 (1967).
[15] C. N. Yang, Selected Papers, 1945-1980, with Commentary (Freeman, San Francisco, CA, 1983), p. 430.

[16] M. Takahashi, Prog. Theor. Phys. 44, 899 (1970).

[17] J. N. Fuchs, A. Recati, and W. Zwerger, Phys. Rev. Lett. 93, 090408 (2004).

[18] S. Sachdev, Quantum Phase Transitions (Cambridge University Press, Cambridge, 1999).

[19] C.-L. Huang et al., Nature (London) 470, 238 (2011).

[20] Q. Zhou and T.-L. Ho, Phys. Rev. Lett. 105, 245702 (2010).

[21] K. R. A. Hazzard and E. J. Mueller, e-print arXiv:1006.0969.

[22] X. W. Guan and T.-L. Ho, e-print arXiv:1010.1301.

[23] E. Zhao, X. W. Guan, W. V. Liu, M. T. Batchelor, and M. Oshikawa, Phys. Rev. Lett. 103, 140404 (2009).

[24] X. W. Guan and M. T. Batchelor, J. Phys. A 44, 102001 (2011).

[25] M. Takahashi, Thermodynamics of One-Dimensional Solvable Models (Cambridge University Press, Cambridge, 1999).

[26] C. N. Yang and C. P. Yang, J. Math. Phys. 10, 1115 (1969).

[27] E. Mueller (private communication).

[28] The correlation length diverges at quantum criticality but is typically smaller than the trapping size [20,21]. Finite-size effects are not essential in an elongated trap; see X. Zhang et al., New J. Phys. 13, 045011 (2011). 